\title{
Quantitative optical coherence tomography angiography of the peripapillary circulation in glaucoma
}

\author{
Joseph M. Simonett, David Huang, and Yali Jia \\ Casey Eye Institute, Oregon Health and Science University, Portland, OR, USA
}

The cause of glaucoma, a disease defined by degeneration of retinal ganglion cells associated with cupping of the optic nerve head $(\mathrm{ONH})$ and progressive vision loss, continues to be debated despite numerous advances in ophthalmic imaging and diagnostics. Although elevated intraocular pressure is often implicated, many studies, using a variety of imaging techniques including plain fundus photography, fluorescein angiography, laser Doppler flowmetry, and color Doppler ultrasound (1-4), have suggested that insufficiencies of ONH circulation may play a role in glaucoma pathogenesis. While all of these imaging modalities have been informative, each has a set of limitations, including low spatial resolution, invasiveness, restriction to superficial vessels, lack of repeatability, and modality specific artifacts. Optical coherence tomography angiography (OCTA), an imaging technology that can produce high resolution, three-dimensional images of fundus microcirculation, is well suited to overcome many of these limitations (5).

In 2012, Jia et al. first described the use of OCTA in quantifying ONH perfusion (6). In this proof of concept study, OCT imaging using the split-spectrum amplitude-decorrelation angiography (SSADA) algorithm was used to measure local microcirculation in the peripapillary regions, and demonstrated a reduction in ONH perfusion in a small cohort of early glaucoma patients. The amplitude-based method of SSADA is sensitive to flow in all directions, making quantification independent of the angle of incidence between OCT beam and direction of blood flow. Furthermore, amplitude decorrelation, as compared to amplitude variance, is less affected by signal strength and allows for greater reliable comparisons between subjects. These factors make SSADA a useful tool for quantifying retinal microcirculation and studying glaucoma related vascular changes.

A recent study by Yarmohammadi et al. investigated the relationship between vessel density, as measured with OCTA, and severity of visual field loss in glaucoma (7). The authors measured peripapillary vessel density in eyes of healthy subjects, glaucoma suspects, mild

Correspondence to: Yali Jia. Casey Eye Institute, Oregon Health \& Science University, 3375 SW Terwilliger Blvd., Portland, OR 97239-4197, USA. jiaya@ohsu.edu.

Provenance: This is a Guest Editorial commissioned by Section Editor Ji-Guo Yu, PhD (Department of Ophthalmology, the Central Hospital of Wuhan, Wuhan, China).

Comment on: Yarmohammadi A, Zangwill LM, Diniz-Filho A, et al. Relationship between Optical Coherence Tomography Angiography Vessel Density and Severity of Visual Field Loss in Glaucoma. Ophthalmology 2016;123:2498-2508.

Conflicts of Interest: Oregon Health \& Science University (OHSU), David Huang, and Yali Jia have a significant financial interest in Optovue, Inc., a company that may have a commercial interest in the results of this research. David Huang received royalties on an optical coherence tomography patent licensed to Carl Zeiss Meditec, Inc. These potential conflicts of interest have been reviewed and managed by OHSU. Joseph M. Simonett has no conflicts of interest to declare. 
(visual field mean deviation $>-6 \mathrm{~dB}$ ) and moderate to severe (visual field mean deviation < $-6 \mathrm{~dB}$ ) primary open-angle glaucoma (POAG) patients. They reported a stepwise qualitative and quantitative loss in microcirculation in each more severely affected patient group, and a relatively strong association between peripapillary vessel density and glaucomatous visual field damage $\left(\mathrm{R}^{2}=0.54\right)$. Interestingly, vascular-functional correlations were stronger than structural (RNFL thickness and optic nerve rim area)-functional correlations, and when multivariate analysis adjusted for these measurements of structural damage, vessel density remained independently associated with visual field loss.

Yarmohammadi et al. limited their vessel density calculations to the superficial vascular plexus contained between the internal limiting membrane and posterior boundary of the RNFL, whereas other studies have measured vessel density in the full thickness retinal slab $(8,9)$. Both of these methods successfully avoid the well described problem of projection artifact, where moving blood cells within overlying vessels cast shadows onto deeper structures, which is misinterpreted as blood flow by the OCTA algorithm (10). While studying the deeper layers of perfusion in isolation is of interest, particularly as some have suggested that the posterior ciliary artery microcirculation supplies the initial site of glaucoma ONH pathology (11), obtaining meaningful OCTA data is limited by projection artifact. In another recent publication from the same group, focal dropout of the peripapillary choroidal microvasculature was demonstrated in glaucomatous eyes, and was spatially associated with lamina cribrosa defects (12). The authors should be commended for strictly defining vascular drop out as a complete loss of decorrelation single, rather than accepting a less stringent threshold of a partial, quantitative decrease in deep-layer microvascular density, as this increases our confidence that identified areas have true flow loss. However, they correctly acknowledge that projection artifact from superficial vessels could result in false-positive detection of blood flow and incorrect classification in the study. Isolation of deep layer circulation within the optic disc, as compared to the peripapillary region, is even more problematic due to the complex anatomy of highly reflective structures. Projectionresolved OCTA, or other correction methods, may be capable of providing a solution to these problems in future studies (13). An additional consideration when interpreting OCTA data is the effect of local OCT reflectance signal strength, which can be weakened by a number of factors including vitreous opacities and pupil edge vignetting resulting in decreased decorrelation signal. Adjustment for reflectance variation has been shown to increase vessel density measurement reliability in OCTA and should be incorporated into future studies (14).

In addition to POAG, other types of glaucoma have recently been studied with OCTA. Scripsema et al. demonstrated a correlation between peripapillary vessel density and severity of glaucomatous vision loss in both POAG and normal tension glaucoma (9). Furthermore, the authors introduce the concept of generating color-coded capillary density maps with OCTA data, which, much like the familiar topographic maps used by clinicians to track RNFL thickness, may prove useful in quickly and objectively evaluating RPC density loss in glaucoma patients. Primary angle-closure glaucoma (PACG), which remains the predominate form of glaucoma in East Asia, has also been investigated. In one study, the diagnostic ability of peripapillary vessel density parameters of OCTA was found to be very similar for both PACG and POAG, and comparable to RNFL thickness measurements (15). 
In another group of PACG eyes, OCTA vessel density findings showed that perfusion of and near the optic nerve were more sensitive to intraocular pressure than elsewhere in the retina (15). These studies highlight the potential OCTA has in comparing and contrasting the underlying mechanisms of different glaucoma subtypes.

In a disease such as glaucoma, where the underlying cause remains unknown, physicians rely on clinical associations to predict disease progression and inform treatment decisions. At the moment, associations of IOP and structural changes, as determined by physical exam and structural imaging, with glaucomatous vision loss are the principal tools in the treating physician's toolbox. With the observational findings of Yarmohammadi et al. and others, the association between glaucoma and impaired $\mathrm{ONH}$ perfusion is strongly supported. Longitudinal studies will help determine the temporal relationship between perfusion changes and disease progression, and to define the clinical role of OCTA in measuring the rate and severity of vessel density loss in glaucoma patients.

\section{Acknowledgements}

Funding: This work was supported by grant DP3 DK104397, R01 EY024544, R01 EY023285, P30 EY010572 from the National Institutes of Health (Bethesda, MD, USA), and by unrestricted departmental funding from Research to Prevent Blindness (New York, NY, USA).

\section{References}

1. Jonas JB, Nguyen XN, Naumann GO. Parapapillary retinal vessel diameter in normal and glaucoma eyes. I. Morphometric data. Invest Ophthalmol Vis Sci 1989;30:1599-603. [PubMed: 2745000]

2. Wolf S, Arend O, Sponsel WE, et al. Retinal hemodynamics using scanning laser ophthalmoscopy and hemorheology in chronic open-angle glaucoma. Ophthalmology 1993;100:1561-6. [PubMed: 8414416]

3. Nicolela MT, Hnik P, Drance SM. Scanning laser Doppler flowmeter study of retinal and optic disk blood flow in glaucomatous patients. Am J Ophthalmol 1996;122:775-83. [PubMed: 8956631]

4. Galassi F, Nuzzaci G, Sodi A, et al. Color Doppler imaging in evaluation of optic nerve blood supply in normal and glaucomatous subjects. Int Ophthalmol 1992;16:273-6. [PubMed: 1428556]

5. Jia Y, Tan O, Tokayer J, et al. Split-spectrum amplitude-decorrelation angiography with optical coherence tomography. Opt Express 2012;20:4710-25. [PubMed: 22418228]

6. Jia Y, Morrison JC, Tokayer J, et al. Quantitative OCT angiography of optic nerve head blood flow. Biomed Opt Express 2012;3:3127-37. [PubMed: 23243564]

7. Yarmohammadi A, Zangwill LM, Diniz-Filho A, et al. Relationship between Optical Coherence Tomography Angiography Vessel Density and Severity of Visual Field Loss in Glaucoma. Ophthalmology 2016;123:2498-508. [PubMed: 27726964]

8. Liu L, Jia Y, Takusagawa HL, et al. Optical Coherence Tomography Angiography of the Peripapillary Retina in Glaucoma. JAMA Ophthalmol 2015;133:1045-52. [PubMed: 26203793]

9. Scripsema NK, Garcia PM, Bavier RD, et al. Optical Coherence Tomography Angiography Analysis of Perfused Peripapillary Capillaries in Primary Open-Angle Glaucoma and Normal-Tension Glaucoma. Invest Ophthalmol Vis Sci 2016;57:OCT611-OCT620. [PubMed: 27742922]

10. Spaide RF, Fujimoto JG, Waheed NK. Image artifacts in optical coherence tomography angiography. Retina 2015;35:2163-80. [PubMed: 26428607]

11. Hayreh SS. The 1994 Von Sallman Lecture. The optic nerve head circulation in health and disease. Exp Eye Res 1995;61:259-72. [PubMed: 7556490]

12. Suh MH, Zangwill LM, Manalastas PI, et al. Deep Retinal Layer Microvasculature Dropout Detected by the Optical Coherence Tomography Angiography in Glaucoma. Ophthalmology 2016;123:2509-18. [PubMed: 27769587] 
13. Zhang M, Hwang TS, Campbell JP, et al. Projection-resolved optical coherence tomographic angiography. Biomed Opt Express 2016;7:816-28. [PubMed: 27231591]

14. Gao SS, Jia Y, Liu L, et al. Compensation for Reflectance Variation in Vessel Density Quantification by Optical Coherence Tomography Angiography. Invest Ophthalmol Vis Sci 2016;57:4485-92. [PubMed: 27571015]

15. Rao HL, Kadambi SV, Weinreb RN, et al. Diagnostic ability of peripapillary vessel density measurements of optical coherence tomography angiography in primary open-angle and angleclosure glaucoma. Br J Ophthalmol 2016 [Epub ahead of print]. 